\title{
Performance Evaluation of UDP, DCCP, SCTP and TFRC for Different Traffic Flow in Wired Networks
}

\author{
Ali Hussein Wheeb \\ Al-Khwarizmi College of Engineering, University of Baghdad, Iraq
}

\begin{tabular}{l}
\hline \hline Article Info \\
\hline Article history: \\
Received Feb 17, 2017 \\
Revised Jun 23, 2017 \\
Accepted Jul 14, 2017 \\
\hline
\end{tabular}

\section{Keyword:}

UDP

DCCP

SCTP

TFRC

Network simulator NS-2

performance evaluation

\begin{abstract}
The demand for internet applications has increased rapidly. Providing quality of service (QoS) requirements for varied internet application is a challenging task. One important factor that is significantly affected on the QoS service is the transport layer. The transport layer provides end-to-end data transmission across a network. Currently, the most common transport protocols used by internet application are TCP (Transmission Control Protocol) and UDP (User Datagram Protocol). Also, there are recent transport protocols such as DCCP (data congestion control protocol), SCTP (stream congestion transmission protocol), and TFRC (TCP-friendly rate control), which are in the standardization process of Internet Engineering Task Force (IETF). In this paper, we evaluate the performance of UDP, DCCP, SCTP and TFRC protocols for different traffic flows: data transmission, video traffic, and VOIP in wired networks. The performance criteria used for this evaluation include throughput, end to end delay, and packet loss rate. Well-known network simulator NS-2 used to implement the UDP, DCCP, SCTP, and TFRC protocols performance comparison. Based on the simulation results, the performance throughput of SCTP and TFRC is better than UDP. Moreover, DCCP performance is superior SCTP and TFRC in term of end-to-end delay.
\end{abstract}

Copyright (C) 2017Institute of Advanced Engineering and Science. All rights reserved.

\section{Corresponding Author:}

Ali H. Wheeb,

Al-Khwarizmi College of Engineering,

University of Baghdad,

Baghdad, Iraq.

Ali.h@kecbu.uobaghdad.edu.iq

\section{INTRODUCTION}

A wired network is a network which connected through physical wires with each other. Data processing and Sharing of data is the main function of the computer network. The Internet provides communication and share data online as the basic service. Currently, the internet has to support varied applications with different requirements. Delay and jitter are essential requirements for application such as video streaming and VoIP service. On the other hand, reliability is a necessary requirement for data transfer application. Therefore, the choice of transport layer protocol depends on the application requirements in term of quality of service.

Nowadays, most of the internet application uses the two main protocols of transport layer transmission control protocol (TCP) and user datagram protocol (UDP). TCP provides a reliable in-order delivery of the data, whereas UDP provides faster delivery of packets as compared to TCP, but it does not provide any congestion control mechanism [1]. Recently, new transport protocols have been designed to call Data Congestion Control Protocol (DCCP), Stream Control Transmission Protocol (SCTP), and TCP-friendly rate control (TFRC). These recent protocols are still under research whether they can be used for real-time application practically. 
The aim of this paper is Evaluating the performances of four Transport layer protocols UDP, DCCP, SCTP, and TFRC in a wired network environment for three traffic flows video traffic, data traffic, and VoIP traffic. Through simulations, the performance of UDP, DCCP, SCTP, and TFRC protocols has been analyzed to say in which scenario the protocol works better than the other. Several studies focus on transport protocols performance. In [2] The three standard protocols TCP, UDP, and TFRC are simulated and performance compared in wired networks. First TCP, UDP, and TRFC are simulated independently and then interoperation of TCP-UDP, TCP-TFRC, and UDPTFRC is studied. In [3] compared the performance of the two main protocols TCP and UDP in the wired network. Network Simulator (NS-2) has been used for performance Comparison since it is preferred by the networking research community. In [4] evaluated the performance of three transport layer protocols UDP, SCTP, and DCCP for transporting MPEG-4 video traffic over Wi-MAX. In [5] compared the protocol behavior of DCCP (Datagram Congestion Control Protocol) with mostly used protocol TCP. Also, describes the basic principle of DCCP, its congestion control mechanism, and measures the performance of DCCP. In [6] evaluated the performance of DCCP/CCID4 on a live satellite link for a number of scenarios which include different voice codecs and a varying number of simultaneous VoIP calls. In [7] authors evaluate the performance of TFRC with modified TFRC over wired networks. Authors in [8] simulated and compared the performance of modified TCP with classical TCP using NS2.

\section{TRANSPORT LAYER PROTOCOLS}

In the TCP/IP model, the transport layer accepts the data from the application layer and adds its header, then forwards to the lower layers for further processing. The transport layer provides efficient, reliable services such as reliable data transfer, congestion control, buffering, flow control and multiplexing /demultiplexing. Therefore, its performance directly affects the application performance as perceived by the user. Many protocols have been proposed by IETF to meet the requirements of the transport layer, the most known are User Datagram Protocol (UDP) [9], Transmission Control Protocol (TCP) [10], Datagram congestion control protocol (DCCP) [11], Stream Control Transmission Protocol (SCTP) [12], and TCP Friendly Rate Control (TFRC) [13].

UDP is one of the basic protocols of internet protocol stack. It is a simple transport layer protocol that does not provide any reliability and in-order delivery of the data packets. Also, there is no congestion control mechanism in UDP. It is very suitable for applications that prefer packet loss to jitter or time critical requirements. UDP is considered where the in time delivery of data is important rather than reliable delivery. So, most of the multimedia application such as video streaming use UDP as their transport protocol. DCCP is a new transport layer protocol proposed by IETF that is providing a message oriented, reliable connection setup, congestion control and feature negotiation. It is designed for those applications where timing constraints exist in the delivery of data but does not require reliable ordered delivery. However, DCCP does not provide any congestion control service at the application layer. It has built in congestion control mechanism. Three congestion control mechanisms of DCCP are TCP-like (CCID 2), TCP-friendly (CCID 2), and TCP-Friendly Rate Control for Small Packets (CCID 4). A congestion control mechanisms can be selected during connection setup and dynamically changed. Practically, this is useful for real time applications where a steady rate of data transmission is required rather than reliable delivery of packets.

SCTP is a recent transport Layer protocol, serving in a similar role as the most prominent protocols: TCP and UDP. In fact, it provides service of both guarantees reliable and in-sequence transport of messages with congestion control mechanisms like TCP, and preserving data message boundaries similar to UDP. SCTP was developed by the IETF Signalling Transport (SIGTRAN) working group with the aim of overcoming limitations of TCP. Therefore, it offers such advantages as multi-homing and multi-streaming capabilities unlike to TCP and UDP. TFRC is also a new transport protocol, that provides a congestion control mechanism for unicast flows operating in a best effort Internet environment. It is reasonably fair when competing for bandwidth with TCP traffic flows, Yet it has a lower variation of throughput over time compared with TCP. Due to this reason, it more suitable for real-time applications such as streaming media where a relatively smooth sending rate is of importance.

\section{RESEARCH METHOD}

Three simulation scenarios have been done in this research for the evaluation of UDP, DCCP, STCP, and TFRC in wired network environments for different traffic flow. Scenarios simulated are listed below:

1. Data traffic over UDP, DCCP, SCTP, and TFRC.

2. Video traffic over UDP, DCCP, SCTP, and TFRC. 
3. VoIP traffic over UDP, DCCP, SCTP, and TFRC.

Table 1 show parameters setting of the The three traffic flows.

Table 1. Traffic Parameters Setting

\begin{tabular}{ccc}
\hline Traffic & Data Rate (kbps) & Packet size (bytes) \\
\hline Data & 500 & 1000 \\
Video & 200 & 500 \\
VoIP & 64 & 128 \\
\hline
\end{tabular}

\section{SIMULATION}

The network simulator NS-2, version 2.35 [14] with the patch for SCTP [15] are used to obtain The simulation results presented in this paper.

\subsection{Simulation Environment}

The simulation network topology consists of four nodes created in a wired environment. Specifically, one source node, one destination node, and two router nodes as shown in Figure 1. However, This node is a static position and connect by a bidirectional link. Simulation parameters setting has been used in our research are shown in Table 2.



Figure 1. Simulation network topology

Table 2. Simulation Parameters Setting

\begin{tabular}{cc}
\hline Parameter & Value \\
\hline Simulator & NS-2.35 \\
Source node & 1 \\
Destination node & 1 \\
Router node & 2 \\
Interface & Wired \\
Queue type & Drop Tail \\
Queue size & 10 Packets \\
Transport Protocls & UDP,DCCP,SCTP,TFRC \\
Application traffic & CBR \\
Simulation Time & 50Sec \\
\hline
\end{tabular}

\subsection{Performance Metrics}

Three performance metrics [16] have been evaluated and analyzed in this study for various Transport Layer Protocols like UDP, SCTP, and DCCP. These performance metrics are described as below:

Throughput Define as the total number of bytes successfully received at the destination in a given amount of time, measured in Kbps. The amount of time may be the Simulation run time or maybe the time between the last packet received and first packet received. End-to-End delay is the time spent for a packet to be transmitted from source to destination across the network, measured in seconds. Furthermore, it is an important criterion to evaluate QoS for real-time application. The performance of network very affected by 
the number of packet loss. The ratio between the number of packets lost to the total number of packets sent define as a packet loss ratio.

\section{RESULTS AND ANALYSIS}

This section described the results of transport layer protocols UDP, DCCP, SCTP, and TFRC obtained from our simulation experiments in different scenarios. Throughput for data traffic, video stream, and VoIP traffic over four transporting protocols are shown in Figure 2, Figure 3, and Figure 4 respectively.

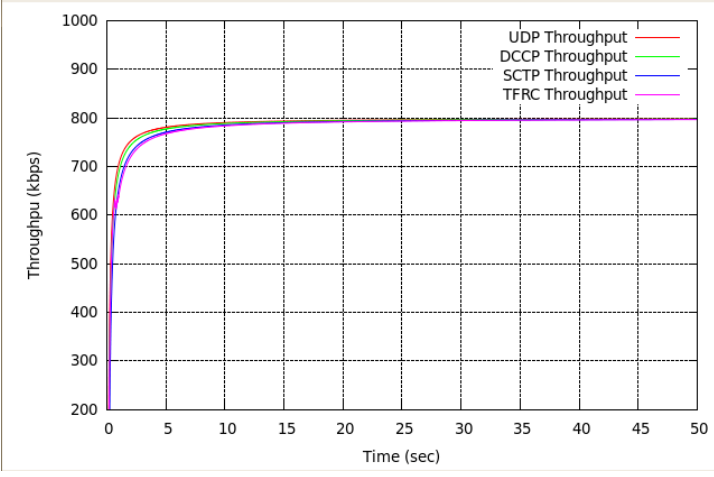

Figure 2. Comparison of throughput using data traffic

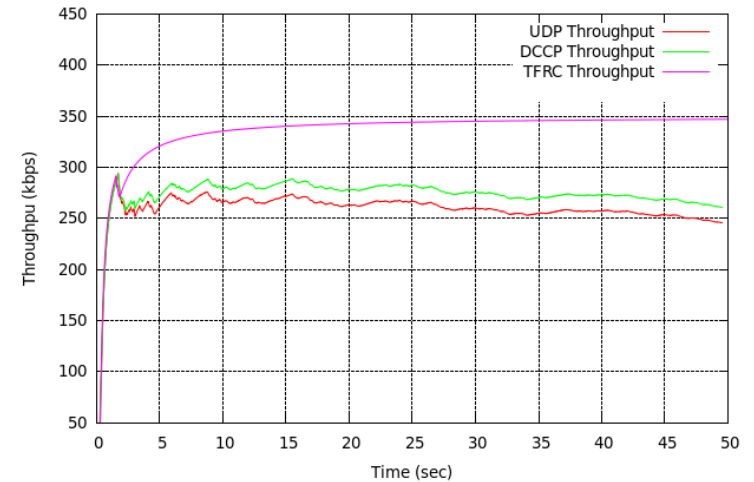

Figure 3. Comparison of throughput using video traffic

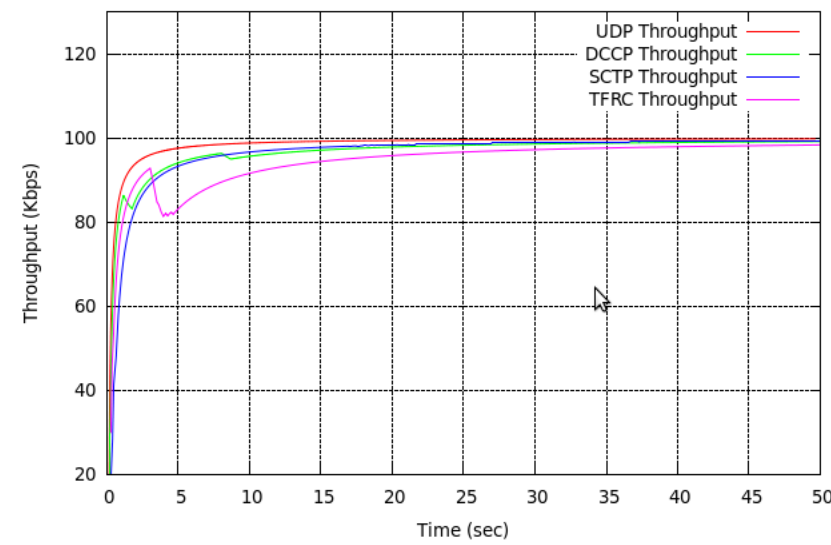

Figure 4. Comparison of throughput using VoIP traffic

From the simulation results, we notice that the throughput of SCTP is a little better than UDP, DCCP, TFRC for data traffic. This is due to that SCTP uses features like multi-streaming and multi-homing. Whereas, TFRC achieved maximum throughput for video streaming. The resulting throughput under SCTP is greater than other protocols in VoIP traffic scenario. Additionally, Table 3 presents a comparison of the average throughput for all traffic flow. For applications that require high throughput and reliable transport, it is suitable to use SCTP and TFRC.

Table 3. Average Trhoughput Comparision

\begin{tabular}{ccccc}
\hline Traffic & $\begin{array}{c}\text { UDP } \\
\text { Average Throughput } \\
(\mathrm{kbps})\end{array}$ & $\begin{array}{c}\text { DCCP } \\
\text { Average Throughput } \\
(\mathrm{kbps})\end{array}$ & $\begin{array}{c}\text { SCTP } \\
\text { Average Throughput } \\
(\mathrm{kbps})\end{array}$ & $\begin{array}{c}\text { TFRC } \\
\text { Average Throughput } \\
(\mathrm{kbps})\end{array}$ \\
\hline Data & 798.84. & 799.508 & 800.162 & 798.846 \\
Video & 246.92. & 262.004 & 326.848 & 348.734 \\
VoIP & 99.5634 & 99.3711 & 100.026 & 98.8558. \\
\hline
\end{tabular}


Figure 5, Figure 6, and Figure 7 illustrates the measured end-to-end delay for different traffic flows over UDP, DCCP, SCTP, and TFRC protocols. The performance of end-to-end delay for DCCP is shorter than other transporting protocols in the data traffic scenario. This is due to that DCCP has a congestion control mechanism hence give less delay. While in video traffic experiment UDP obtains the minimal end-toend delay. The TFRC results in lower overall end-to-end delay for UDP, DCCP, and SCTP for VoIP traffic.

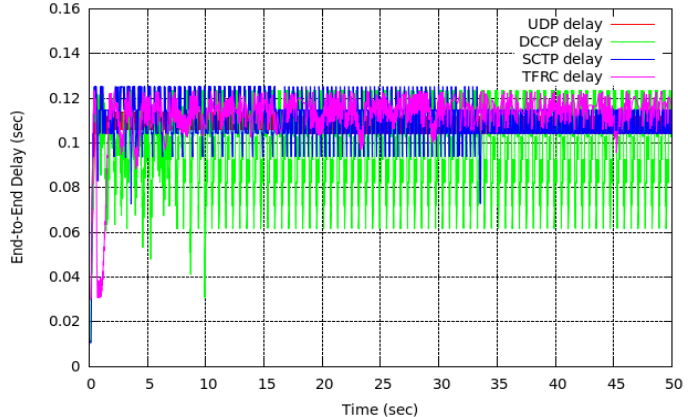

Figure 5. End-to-End delay of UDP, SCTP, DCCP, TFRC for data traffic

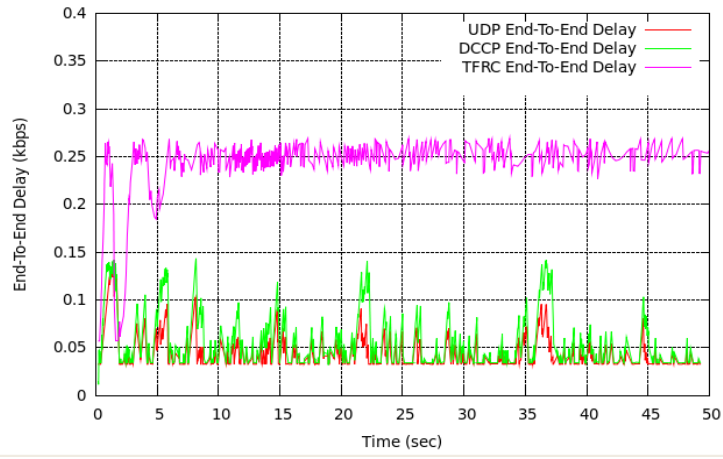

Figure 6. End-to-End delay of UDP, SCTP, DCCP, TFRC for video traffic

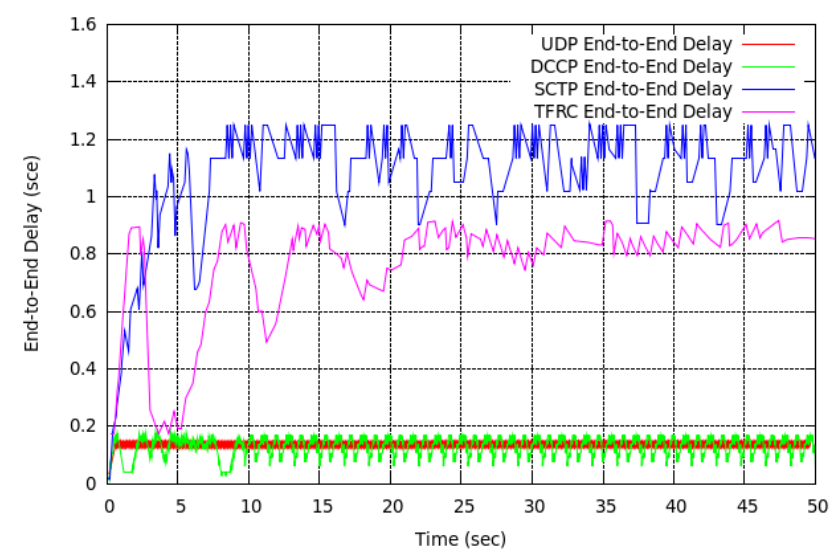

Figure 7. End-to-End delay of UDP, SCTP, DCCP, TFRC for VoIP traffic

End-to-End delay is a very important issue for real-time application. A comparison of average endto-end delay of UDP, DCCP, SCTP, and TFRC for three traffic flows is shown in Table 4.

Table 4. Average Delay Comparision

\begin{tabular}{ccccc}
\hline Traffic & $\begin{array}{c}\text { UDP } \\
\text { Average Delay } \\
(\mathrm{sec})\end{array}$ & $\begin{array}{c}\text { DCCP } \\
\text { Average Delay } \\
(\mathrm{sec})\end{array}$ & $\begin{array}{c}\text { SCTP } \\
\text { Average Delay } \\
(\mathrm{sec})\end{array}$ & $\begin{array}{c}\text { TFRC } \\
\text { Average Delay } \\
(\mathrm{sec})\end{array}$ \\
\hline Data & 0.108356 & 0.0992315 & 0.111009 & 0.113008 \\
Video & 0.0467266 & 0.0596024 & 0.13752 & 0.24634 \\
VoIP & 0.136904 & 0.128799 & 1.07451 & 0.783358 \\
\hline
\end{tabular}

Table 5 reflect The packet loss ratio comparison between UDP, DCCP, SCTP, and TFRC for different traffic flows. The result shows that the TFRC packet loss ratio is lower than other transporting protocols in the first scenario. This due to the friendly behavior and steady transmission rate. The performance of packet loss for DCCP is better than UDP, SCTP, and TFRC in VoIP Traffic. Based on Table 5, SCTP performance is better for packet loss ratio than UDP, DCCP, and TFRC in video stream cases. However, the performance of DCCP for packet loss is nearly slight. 
Table 5. Packet Loss Ratio Comparison

\begin{tabular}{ccccc}
\hline Traffic & $\begin{array}{c}\text { UDP } \\
\text { Packet Loss Ratio } \\
(\%)\end{array}$ & $\begin{array}{c}\text { DCCP } \\
\text { Packet Loss Ratio } \\
(\%)\end{array}$ & $\begin{array}{c}\text { SCTP } \\
\text { Packet Loss Ratio } \\
(\%)\end{array}$ & $\begin{array}{c}\text { TFRC } \\
\text { Packet Loss Ratio } \\
(\%)\end{array}$ \\
\hline Data & 11.03 & 1.73543 & 3.00791 & 1.41559 \\
Video & 0.328407 & 0.172191 & 0.1376 & 1.68561 \\
VoIP & 12.1835 & 1.9996 & 2.51046 & 2.00777 \\
\hline
\end{tabular}

\section{CONCLUSION}

Recent and standard transport layer protocols effect directly on internet application service since it transports data traffic over networks. In this paper, we have simulated and evaluated the performance of UDP, DCCP, SCTP, and TFRC as the transport protocol for three traffic flows in wired networks. The four transport protocols are implemented using NS-2. Data traffic flow, video stream flow, and VoIP traffic flow are transported respectively. The simulation result showed that SCTP can achieve the best of the performance throughput except video stream traffic where TFRC performance is better. However, less end-to-end delay obtained by DCCP in the data traffic transmission. Whilst, UDP has a lower delay for video streaming flow.

\section{REFERENCES}

[1] S. Kumar and S. Rai, "Survey on Transport Layer Protocols: TCP \& UDP”, International Journal of Computer Applications, vol. 46, pp. 20-25, May 2012.

[2] C.R. Pakanati, M. Padmavathamma, and N.R. Reddy, "Performance Comparison of TCP, UDP, and TFRC in Wired Networks", 2015 IEEE International Conference on Computational Intelligence \& Communication Technology, pp. 257-263, 2015.

[3] Ali H. Wheeb, "Performance Comparison of Transport Layer Protocols", International Journal of Advanced Research in Computer Science and Software Engineering, vol. 5, pp. 121-125, Dec 2015.

[4] H. Muhammad, O. Chughtai, S. A. Malik, M. Yousaf, "Performance Evaluation of Transport Layer Protocols for Video Traffic over WiMax", IEEE, 2009.

[5] I.S. Chowdhury, J. Lahiry, K.C. Rahman, and S.F. Hasan, "Performance Analysis of Datagram Congestion Control Protocol (DCCP)", International Journal of Computer Theory and Engineering, vol. 3, pp. 632-637, Oct 2011.

[6] G. Sarwar, R. Boreli and E. Lochin, "Performance of VoIP with DCCP for Satellite Links", 2009 IEEE International Conference on Communications, July 2009.

[7] N.R. Reddy, C.R. Pakanati, and M. Padmavathamma, "Performance Enhancement of TCP Friendly Rate Control Protocol over Wired Networks", International Journal of Electrical and Computer Engineering (IJECE), vol. 6, pp. 2949-2954, Dec 2016.

[8] S.S. Arefin, I. Azad, H. Kabir, "Modified SACK-TCP and some Application Level Techniques to Support realTime Application", International Journal of Electrical and Computer Engineering (IJECE), vol. 6, pp. 105-114, may 2013

[9] J. Postel, "User Datagram Protocol”, Internet Engineering Task Force (IETF), vol. 768, Aug 1980.

[10] J. Postel, "Transmission Control Protocol”, Internet Engineering Task Force (IETF), vol. 793, Sep 1981.

[11] E. Kohler, M. Handley, and S. Floyd, "Datagram Congestion Control Protocol (DCCP)", Internet Engineering Task Force (IETF), vol. 4340, Mar 2006.

[12] R. Stewart, "Stream Control Transmission Protocol (SCTP)", Internet Engineering Task Force (IETF), vol. 4960, Sep 2007.

[13] M. Handley, S. Floyd, J. Padhye, and J. Widmer, "TCP Friendly Rate Control (TFRC): Protocol Specification", Internet Engineering Task Force (IETF), vol. 5348, Jan 2008.

[14] Network Simulator- NS2- http://www.isi.edu/nsnam/ns.

[15] SCTP Patch for NS. http://pel.cis.udel.edu

[16] S. Floyd, "Metrics for the Evaluation of Congestion Control Mechanisms", Internet Engineering Task Force (IETF), vol. 5166, Mar 2008.

\section{BIOGRAPHY OF AUTHOR}



Ali H. Wheeb received a M.Sc degree in Computer Engineering from the university of Baghdad, Baghdad, Iraq, in 2014, and B.Sc degree in Control and System engineering from the University of Technology, Baghdad, Iraq, in 2007. His research interests include Transport Layer protocols, Wireless Sensor Networks (WSN), and Mobile Ad-hoc Network (MANET). Currently, he is a Lecturer and Director the Mechatronics Department of University of Baghdad, Iraq. 